\title{
Solvation dynamics in liquid water. I. Ultrafast energy fluxes
}

\author{
Rossend Rey* \\ Departament de Física i Enginyeria Nuclear, Universitat Politècnica \\ de Catalunya, Campus Nord B4-B5, Barcelona 08034, Spain.
}

\author{
James T. Hynes ${ }^{\dagger}$ \\ Department of Chemistry and Biochemistry University of Colorado, Boulder, CO 80309-0215 USA, \\ Chemistry Department, Ecole Normale Supérieure, \\ UMR ENS-CNRS-UPMC 8640, 24 Rue Lhomond, 75005 Paris, France
}

\begin{abstract}
Solvation dynamics in liquid water is addressed via nonequilibrium energy transfer pathways activated after a neutral atomic solute acquires a unit charge, either positive or negative. It is shown that the well-known nonequilibrium frequency shift relaxation function can be expressed in a novel fashion in terms of energy fluxes, providing a clearcut and quantitative account of the processes involved. Roughly half of the initial excess energy is transferred into hindered rotations of first hydration shell water molecules, i.e. librational motions, specifically those rotations around the lowest moment of inertia principal axis. After integration over all water solvent molecules, rotations account for roughly $80 \%$ of the energy transferred, while translations have a secondary role; transfer to intramolecular water stretch and bend vibrations is negligible. This picture is similar to that for relaxation of a single vibrationally or rotationally excited water molecule in neat liquid water, although solvation relaxation is more non-local. In addition, we find a remarkable independence of the main relaxation channels on the newly created charges sign. Although the methodology is applied here to the simplest solute case, the approach is rather general, and it should be at least equally useful in more realistic and complex scenarios.
\end{abstract}

Keywords: Energy transfer, frequency shift, librations, ion hydration, power, work.

\section{INTRODUCTION}

Solvation dynamics or relaxation, i.e. the dynamic response of a surrounding solvent (or more generally an environment) to a charge redistribution of a solute upon its electronic excitation, has attracted considerable attention over the years, both experimentally and theoretically ${ }^{1-9}$. From the experimental standpoint, its study has mostly centered up to now on the electronic excitation of fluorescent probes ${ }^{10-12}$, which generally entails charge redistribution on the solute. The ensuing time dependent frequency shift encapsulates the complex dynamics that results from free energy minimization of the solvent to the new solute configuration. While originally focused on relatively simple solutes in comparatively simple environments, the study of solvation dynamics has now spread to a panoply of situations including - among others - electrolyte solutions ${ }^{13,14}$, supercritical fluids ${ }^{15-17}$, ionic liquids ${ }^{18,19}$, liquid interfaces ${ }^{20,21}$, micelles $^{22,23}$, hydrophobic cavities ${ }^{24}$, proteins $^{25-30}$, and $\mathrm{DNA}^{31-34}$.

Among the initial rationales for the study of solvation dynamics was the determination of solvation time scales and the connection of those dynamics to the rates of chemical reactions. The closest solvation dynamicsreaction rate connection is for low barrier electron ${ }^{2,5,35,36}$ and proton transfers ${ }^{37}$, with a partial connection for other reaction classes ${ }^{38-40}$. There is however another potential solvation dynamics connection which is relatively little explored, i.e. if the solvent relaxation were to be analyzed in terms of energy flow or transfer into solvent degrees of freedom, this could provide information useful for comprehending chemical reaction microscopic mechanisms or reaction paths, beyond the more limited issue of the reaction rate ${ }^{41}$. Indeed, the channeling of energy in chemical reaction pathways depends critically on translational, rotational and vibrational modes of the solvent. In the present paper, we illustrate the development of such an energy flow perspective for solvation dynamics.

Unfortunately, the information provided by solvation dynamics experiments is generally limited to the time scales involved. As a result, numerical simulation has become particularly useful for theoretical interpretation. This is the route we will follow for the application of the energy flow perspective, but before doing that, we briefly discuss the traditional computational approach to the problem.

The most popular computational approach for solvation dynamics, which has often been quite useful in interpreting some of its features, has consisted in ascertaining, most often by inferring, the solvent modes involved in a somewhat indirect way, namely from the equilibrium time correlation function (tcf) of energy gap fluctuations (the energy difference between ground and excited solute states, with the solvent initially in equilibrium with the solute ground state). Comparison of the associated power spectrum with the known spectroscopic features of the solvent has sometimes served to qualitatively identify the solvent motions involved; the situation here is analogous to that for tcf studies of vibrational energy transfer, where similar comparisons implicate e.g. which solvent modes are energy receptors without directly observing the solvent molecules receiving the energy ${ }^{42-45}$. It is to be noted that, besides its qualitative character, this tcf 
approach suffers from additional limitations. Most importantly, it invokes the validity of linear response theory, which is an issue to consider here, given the large perturbations inherent to the creation or alteration of a charge distribution; indeed linear response is not always applicable to the solvation dynamics problem ${ }^{46-49}$. Even when such a treatment does a reasonable job on the overall time dependence, this is no guarantee that detailed analysis involving molecular features within this formulation is completely reliable. Further, even within the framework of linear response theory, there is not a unique way to proceed for this particular problem. Namely, while usually the energy gap time dependence is computed with the solvent in equilibrium with the solute ground state electronic surface, it is at least equally reasonable to compute it with the solute's excited state dynamics ${ }^{50}$. In addition, it is important to note that the qualitative identification of the spectral bands involved requires little band overlap in the accepting solvent modes. This is fortunately the case in water, where translational, librational and vibrational bands are fairly well separated but, even in this case, it does not allow for instance to ascertain which molecular rotations (i.e. which principal axes) are involved, due to their spectral overlap.

Recently, we have shown that, via a power/work formulation, a detailed computation of energy fluxes ${ }^{51}$, as opposed to energy fluctuations (in equilibrium computations) or energy changes (in nonequilibrium computations), constitutes a most convenient method to unravel at the molecular level the energy relaxation processes that follow nonequilibrium excitations of solute vibrational and rotational modes. This approach has provided a fresh and clearcut perspective on the energy pathways for vibrational ${ }^{51,52}$ and hindered rotational relaxation ${ }^{53}$ in neat liquid water. The crucial advantage of this methodology is that it pinpoints which solvent molecules/modes/solvation shells account for the absorption of the excess energy, providing a quantitative computation of the proportions thereof. As we have pointed out ${ }^{51}$, the same approach should prove valuable in the case of solvation relaxation of an electronically excited solute. It will be shown within that the computation of energy fluxes for this problem renders a much more rich picture both qualitatively and quantitatively. It is important to note, in addition, that the energy flow perspective is definitely closer to the experimental situation: as a nonequilibrium approach, it does not require the system to be close to the linear response regime. In fact, the ability of this method to pinpoint the recipients of the energy fluxes can be of help in this connection as well. In particular, it allows a more detailed scrutiny of the differences between equilibrium and nonequilibrium simulations, now in terms of energy fluxes instead of the usual (and more coarse) comparison of energy gap relaxation functions.

Since this is the first instance where the energy flux methodology is applied to solvation dynamics, we focus on the simplest solute system, one that in fact has been a workhorse of the field, namely a neutral monoatomic that suddenly acquires a unit charge (without size change). Indeed, a large part of the present insight on solvation relaxation comes from the study of simple ions ${ }^{15-17,46,48,54-57}$ or dipoles ${ }^{50,58,59}$. We note that in this case, for which the solute is initially neutral, the frequency shift can be directly identified with the Coulomb energy of the solute-solvent interaction ${ }^{50}$ (see below). The computational procedure is thus extremely simple: starting from independent configurations of a neutral solute to which the solvent is equilibrated, a unit charge is placed on the solute and the ensuing transfer of Coulomb energy into the solvent and solute modes is followed and averaged over nonequilibrium trajectories ${ }^{60}$. It is worth stressing that, while the chosen solute and computational procedure are simple, the solvent chosen is water, for which that epithet is hardly applicable. Finally, since we have selected such a well-studied solute situation, there will be a certain overlap of the conclusions of the present work with prior work, especially with the studies by Maroncelli and Fleming ${ }^{46}$, by Tran and Schwartz $^{56}$, and by Bagchi and coworkers ${ }^{61-63}$ on much the same system. In such cases, we indicate what is new or advantageous in our own approach ${ }^{64}$.

We need to add an important word here on the language we will use within for rotations. The water modes in question are in actuality librational motions, i.e. hindered rotations. Unfortunately, no practical description of the librational modes in water is currently available, and we employ the water molecules rotational kinetic energy as the modes for the application of the formulation. We will thus typically employ the nomenclature "rotation" or "rotational", but will occasionally use "librational" in circumstances where it is appropriate.

The outline of the remainder of this paper is as follows. In Sec. II, we present the basic theoretical formulation. Sec. III is devoted to summarizing the computational procedures and parameters used. A detailed discussion of the results obtained is the subject of IV. Concluding remarks are offered in Sec. V.

\section{THEORY}

\section{A. Solute and solvent description}

We first summarize the theoretical construct that allows us to link the experimental frequency shift with the Coulomb energy computed in a numerical simulation. We follow Ref. 50, with minor adjustments, now applied to a simplified model of a monoatomic solute which can be in either of two electronic states: a neutral ground state (denoted with the subscript $n s$ ) and an excited charged state $(c s)$. The Hamiltonians including the solvent are, respectively,

$$
\begin{aligned}
H_{n s} & =K+H_{n s}^{0}+H_{s}+U_{n s, s}, \\
H_{c s} & =K+H_{c s}^{0}+H_{s}+U_{c s, s} .
\end{aligned}
$$


The first two terms correspond in each case to the contribution associated with the unperturbed solute: $K$ denotes the kinetic center of mass translational energy, and $\left(H_{n s}^{0}, H_{c s}^{0}\right)$ are the constant electronic energies in the ground and excited states respectively, so that the unperturbed transition energy is given by

$$
\hbar \omega_{0}=H_{c s}^{0}-H_{n s}^{0} .
$$

The solvent kinetic and potential energies are grouped into the single $H_{s}$ term. Finally, the terms $\left(U_{n s, s}, U_{c s, s}\right)$ correspond to the solute-solvent interactions in the solute ground and excited states respectively. In the present model, the neutral interaction is represented by LennardJones (LJ) interactions between the solvent molecules and the solute. The interaction with the charged state is represented by an additional Coulomb interaction $\left(V_{c s, s}^{c}\right)$, so that the energy gap between both states (for a given solvent configuration) is expressed

$$
\Delta E(t)=H_{c s}-H_{n s}=V_{c s, s}^{c}(t)+\hbar \omega_{0},
$$

which shows that the instantaneous frequency shift $\left(\Delta E(t)-\hbar \omega_{0}\right)$ is given solely by the instantaneous Coulomb energy of the solute-solvent interaction. The average frequency shift $(\delta \hbar \omega)$ can thus be determined as a nonequilibrium average over trajectories (denoted by overbars)

$$
\overline{\delta \hbar \omega(t)}=\overline{V^{c}(t)},
$$

where the subscripts of the Coulomb energy have been dropped for clarity (as will be done whenever no confusion is introduced).

Moreover, the normalized frequency shift, which is the usual focus of interest, can also be expressed in terms of Coulomb energies as well

$$
S(t) \equiv \frac{\overline{\delta \hbar \omega(t)}-\overline{\delta \hbar \omega(\infty)}}{\overline{\delta \hbar \omega(0)}-\overline{\delta \hbar \omega(\infty)}}=\frac{\overline{V^{c}(t)}-\overline{V^{c}(\infty)}}{\overline{V^{c}(0)}-\overline{V^{c}(\infty)}}
$$

In the usual computational approach based on the validity of linear response, this function is identified with the equilibrium time correlation function

$$
C(t)=\frac{\left\langle\delta V^{c}(t) \delta V^{c}(0)\right\rangle}{\left\langle\left(\delta V^{c}(0)\right)^{2}\right\rangle}
$$

where $\delta V^{c}(t) \equiv V^{c}(t)-\left\langle V^{c}\right\rangle$, and the mean values are computed over a long trajectory in the ground state of the solute (i.e. with a neutral monoatomic in the present case). It is here that we see that another possibility is to perform a similar equilibrium calculation with a excited state charged monoatomic which, at least in the case of a model diatomic, results in a somewhat better agreement with $S(t)$.

\section{B. Power and work}

We now turn to the approach applied here, and its connection with the formulas above. As detailed in Refs. 51,52 the motivation for a nonequilibrium approach based on the computation of energy fluxes (power) stems from the simple fact that these fluxes can be disentagled in terms of the contributions from each molecule and its degrees of freedom. This provides a detailed and simple view of the process. In our view, this feature is not shared by formula 7 , in which contributions from different molecules appear highly entangled, making it difficult —or at least not all straightforward - to ascertain the origin of the relaxation in terms of molecular contributions to time correlation functions ${ }^{49,65,68}$.

For our problem, we are interested in the time evolution of the Coulomb potential energy over nonequilibrium trajectories. Starting from an equilibrated neutral solute and solvent, a charge is created, and the solute and solvent subsequently evolve respectively in the presence of the excited solute state. The corresponding Hamiltonian (without constant terms) is

$$
H=K_{\text {solute }}+V_{c s, s}^{c}+V_{c s, s}^{L J}+K_{\text {solvent }}+U_{\text {solvent }},
$$

where the last two terms are the Hamiltonian $H_{s}$ in Eqs. 1,2 .

The time derivative of the quantity of interest (the solute-solvent Coulomb energy) can be simply expressed in terms of Poisson brackets ${ }^{51}$ as

$$
\begin{aligned}
\frac{d V^{c}}{d t} & =\left[V^{c}, H\right]=\left[V^{c}, K_{\text {solute }}\right]+\left[V^{c}, K_{\text {solvent }}\right]= \\
& =-\vec{F}^{c}{ }_{s} \cdot \vec{v}_{s}-\sum_{j} \vec{F}_{n s, j} \cdot \vec{v}_{j},
\end{aligned}
$$

where $\vec{F}_{s}$ denotes the total Coulomb force on the solute, and $\overrightarrow{F C}_{n s, j}$ the corresponding force on each molecular site $(j)$ resulting from the newly created charge on the solute (note that this Coulomb force is not the total Coulomb force acting on the site, as it does not include Coulomb interactions with the rest of the solvent molecules). In short, the important point to note is that the time variation of the Coulomb energy is expressed in terms of a simple sum of energy fluxes (power, $P$ ) into the various sites, i.e.

$$
\frac{d V^{c}}{d t}=-P_{\text {solute }}^{c}-\sum_{j} P_{j}^{c}
$$

While this sum is performed over all molecular sites, depending on the model employed (rigid/flexible water molecules), it can also be expressed in terms of a sum over translational, hindered rotational and vibrational modes (flexible molecules), or just over translations and rotations (rigid molecules), as is done in Section II C.

Of course, for the solvation dynamics/relaxation problem, and as is evident from Eq. 6, we need the integrated 
result $\left(V^{c}(t)\right)$, which is straightforward from Eq. 10,

$$
V^{c}(t)-V^{c}(0)=-W_{\text {solute }}^{c}-\sum_{j} W_{j}^{c} \equiv \Delta V^{c}(t),
$$

i.e. a sum over the works on each site stemming from the solute-solvent Coulomb forces. The increment $\left(\Delta V^{c}(t)\right)$ defined by this formula will constitute our main focus of interest, since it can be easily shown that Eq. 6 can be written

$$
S(t)=\frac{\overline{\Delta V^{c}(t)}}{\overline{V^{c}(0)}-\overline{V^{c}(\infty)}}+1
$$

i.e. $S(t)$ is a normalized and shifted version of $\Delta V^{c}(t)$, so that the latter (not being normalized) contains somewhat more information.

\section{Mode participation in solvation relaxation}

As stated above, the energy fluxes into each one of the molecular sites can be split into the modes of interest, which depend on the solvent molecule models used.

\section{1. $\quad$ Rigid molecules}

In this case, we only need to consider that for each molecule $(i)$, the velocity of a molecular site $\left(i_{a}\right)$ is given by

$$
\vec{v}_{i_{a}}=\vec{v}_{i}^{C M}+\vec{\omega}_{i} \times \vec{r}_{i_{a}}
$$

where $\vec{v}_{i}^{C M}$ denotes the center of mass velocity, $\vec{\omega}_{i}$ is the rotational angular velocity, and $\left(\left\{\vec{r}_{i_{a}}\right\}\right)$ denote the position vectors with respect to the molecular center of mass.

The contribution to the power from each molecule (see Eqs. 9,10) is thus

$$
\begin{aligned}
P_{i} & =\sum_{i_{a}} \vec{v}_{i_{a}} \cdot \vec{F}_{i_{a}}^{c}=\sum_{i_{a}}\left(\vec{v}_{i}^{C M}+\vec{\omega}_{i} \times \vec{r}_{i_{a}}\right) \cdot \vec{F}_{i_{a}}^{c}= \\
& =\vec{v}_{i}^{C M} \cdot \vec{F}_{i}^{c}+\vec{\omega}_{i} \cdot \vec{\tau}_{i}^{c} \equiv P_{i}^{T}+P_{i}^{R},
\end{aligned}
$$

i.e. there is a translational contribution, $P_{i}^{T}$ (where $\vec{F}_{i}^{c}$ denotes the total Coulomb force exerted by the ion), and a rotational contribution, $P_{i}^{R}$ (where $\vec{\tau}_{i}^{c}$ denotes the torque on the solvent molecule exerted by these same forces).

In its integrated form, the function of interest $\left(\Delta V^{c}(t)\right)$ can be now expressed in terms of a sum of works on rotations and translations,

$$
\Delta V^{c}(t)=-W_{\text {solute }}^{T}-\sum_{i} W_{i}^{T}-\sum_{i} W_{i}^{R}
$$

where the first term on the right denotes the Coulomb work on solute translation, while the last two terms denote work on translations and rotations of the solvent molecules, again resulting from the solute-molecule Coulomb interaction. As emphasized in the Introduction, the crucial advantage of this expression stems from the fact that it is clearly partitioned into molecular and mode contributions, i.e. we can unambiguously ascertain the participation of each molecule and whether this contribution is related to its translation and/or rotation.

This approach can be further extended for both translations and rotations. Thus, work on rotations can be partitioned into the contributions from each principal axis, since in the body-fixed frame of reference we can write

$$
\vec{\omega} \cdot \vec{\tau}^{c}=\omega_{x} \cdot \tau_{x}^{c}+\omega_{y} \cdot \tau_{y}^{c}+\omega_{z} \cdot \tau_{z}^{c},
$$

with the definition of axes given in Figure 1. It is important to note that these axes are here ordered according to increasing moment of inertia, as given in the following in terms of the bend angle $(\theta)$ and bond length $(R)$

$$
\begin{aligned}
& I_{x x}=\frac{2 R^{2} m_{O} m_{H}}{M} \cos ^{2}\left(\frac{\theta}{2}\right) \cong 0.6 ; \\
& I_{y y}=2 m_{H} R^{2} \sin ^{2}\left(\frac{\theta}{2}\right) \cong 1.3 ; \\
& I_{z z}=I_{x x}+I_{y y} \cong 1.9
\end{aligned}
$$

where the numbers correspond to the geometry of the rigid model used here (in units amu $\cdot \AA^{2}$ ).

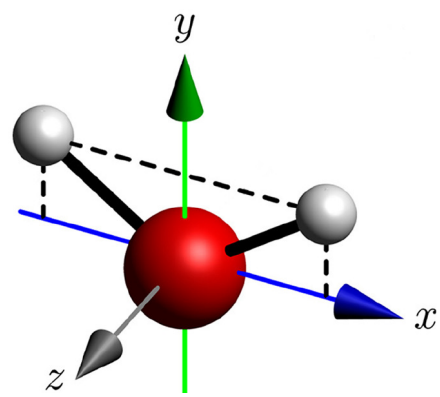

FIG. 1: Definition of axes for the water molecule.

In the case of translations, we can also partition the work into two contributions. The polarizability spectrum following electronic excitation of a dye has been recently measured experimentally for acetonitrile and chloroform solvents ${ }^{69}$. These measures have been partitioned into isotropic and anisotropic components, as defined from appropriate combinations of the experimental signals based on their symmetry. Inspired by these measures, we have partitioned the translational work into that along the ionmolecule axis, which we associate with a isotropic component, and that perpendicular to that axis, and referred to as anisotropic.

\section{Flexible molecules}

In this case, the additional contribution of molecular stretch and bend vibrations needs to be considered. The 
laboratory velocity of a site $\left(i_{a}\right)$ within a given molecule is given by

$$
\vec{v}_{i_{a}}=\vec{v}_{i}^{C M}+\vec{\omega}_{i} \times \vec{r}_{i_{a}}+\vec{v}_{i_{a}}^{v}
$$

where the additional contribution $\left(\vec{v}_{i_{a}}^{v}\right)$ corresponds to the vibrational velocity in the Eckart frame ${ }^{70}$. At each configuration, the center of mass, rotational and vibrational velocities need to be determined from the instantaneous positions and velocities, a procedure detailed in Supporting Information. Once this is accomplished, we obtain again that the total variation of the solvation energy can be written as

$$
\Delta V^{c}(t)=-W_{\text {solute }}^{T}-\sum_{i} W_{i}^{T}-\sum_{i} W_{i}^{R}-\sum_{i} W_{i}^{V},
$$

i.e. we have an additional vibrational contribution compared to the rigid molecule case, Eq. 15. In principle, just as for translations and vibrations, this contribution could be partitioned into the different vibrational modes of the molecules. We choose not to do so, given the negligible contribution of this channel for the water solvent, as shown in Section IVB.

\section{COMPUTATIONAL DETAILS}

We have considered both flexible and rigid models for the water solvent. For the rigid case, the SPC/E model ${ }^{71}$ has been adopted. If a flexible model is used instead, we have followed the same choice as Tran and Schwartz in Ref. 56, namely the SPC/F model due to Toukan and Rahman $^{72}$, where intramolecular flexibility is achieved by adding a phenomenological intramolecular force field to the SPC model. As for the solute, we have again adopted the same choices as Tran and Schwartz ${ }^{56}$ for ease of comparison. For the solute ground state, the water-solute interaction was chosen to be identical to the water-water LJ interaction (for this water model, there are no LJ terms associated with the hydrogens). The excited states, characterized by a positive or negative unit charge, are simply constructed by grafting the corresponding Coulomb interaction on top of the LJ contribution.

All simulations have been run with an in-house code using one solute and 199 water molecules, which for a cut-off distance of half the box length corresponds to an interaction length of $9 \AA$. The Ewald sum correction has been included for Coulomb forces. The integration time step is $1 \mathrm{fs}$ for rigid water molecules and 0.25 fs for flexible molecules. After equilibration, the production simulations consist of a long trajectory from which, every $2.5 \mathrm{ps}$, the instantaneous configuration is taken as the initial one for a separate nonequilibrium run, usually of 2.5 ps length as well, along which the quantities of interest are calculated. Temperature control is maintained ${ }^{73}$ during the equilibration run, and turned off at the start of each non-equilibrium trajectory. Further details will be reported within when required.

\section{RESULTS AND DISCUSSION}

\section{A. Energy gap relaxation function}

Given its central role in solvation relaxation, we start by summarizing the basic features of the nonequilibrium energy gap relaxation function, $S(t)$, defined in Eq. 6 . Fig. 2 displays the result obtained for a flexible solvent. Its behavior is in good agreement with results reported in the past by many groups ${ }^{17,46,55,57,61-63}$. The rationalization of general aspects of this function has been discussed numerous times as well (see the most recent review $^{9}$ for access to the extensive literature here): relaxation is basically bimodal with an initial fast decay (sometimes termed "inertial") ${ }^{74}$ followed by a regime with much slower relaxation (often termed "diffusive"). The respective weight of these regimes has been seen to depend on the solvent. In the present water solvent case, the initial decay (with a time scale of tens of femtoseconds) accounts for the largest portion, as is usually the case for highly dipolar solvents, while the "diffusive" regime is less important, though not negligible, and is characterized by a time scale on the order of a picosecond. In addition, in this particular case (ion solvation), the initial portion of the latter regime is characterized (in simulations) by marked oscillations superimposed on the slow decay.

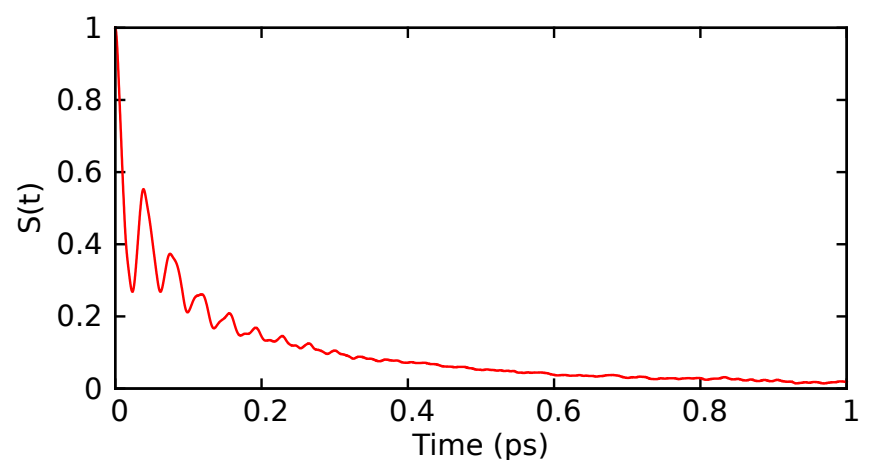

FIG. 2: Nonequilibrium energy gap relaxation function $(\mathrm{S}(\mathrm{t})$, see Eq. 6), for positively charged ion and flexible water solvent.

As described in Section II, for the present problem $S(t)$ is a normalized and shifted version of the Coulomb energy increment $\left(\Delta V^{c}(t)\right)$, which is the raw function computed in simulation. Since this is not normalized, $\Delta V^{c}(t)$ provides additional relevant information, as mentioned in connection with Eq. 12 and as displayed in Figure 3, which includes the result for both rigid and flexible water models (again for a positively charged solute). Their very similar behavior is reassuring, considering the phenomenological nature of the models used (the slightly higher long-term stability shown by the SPCE model can be attributed to the higher site charges). Of probably greater interest is the substantial change in Coulomb solvation energy, $112 \mathrm{kcal} / \mathrm{mol}$, between the initial (nonequi- 
librium) state and the final (equilibrium) state. It is this feature - which of course is extreme for the ionic excited state case - that justifies the concern in connection with the application of linear response theory, both with respect to time scales and any detailed molecular interpretation.

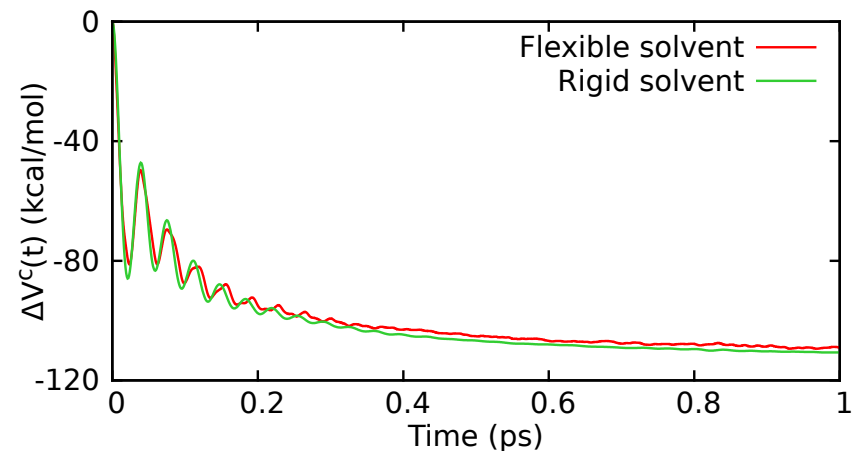

FIG. 3: Coulomb energy increment $\left(\Delta V^{c}(t)\right.$, see Eq. 11), corresponding to a positive ion in rigid (green) or flexible (red) water solvent.

\section{B. Energy fluxes: flexible molecules}

We now proceed to the application of the power/work methodology detailed in section IIB, which for the flexible water solvent produces Eq. 19, relating the Coulomb energy increment (for the creation of a positive charge) discussed in the previous paragraph with the total works exerted on translations, rotations and vibrations. The basic results are displayed in Fig. 4 (corresponding to an average over 800 trajectories, each $2.5 \mathrm{ps}$ long). The picture conveyed (Fig. 4(a)) is remarkably clear: $78 \%$ of the total energy transferred is channeled to rotations (and thus librations) of the neighboring water molecules. This is followed by a much less important transfer into translations of the solvent waters $(18.5 \%)$; ion translations take up $2 \%$, and vibrations come last, barely reaching a $1 \%$ uptake. These results - which are in line with the pioneering, less direct efforts on this system ${ }^{46,56,61-63,75}$ constitute a core finding of the present work since, to the best of our knowledge, they provide the first unambiguous quantitative evidence of the processes at work in water solvation dynamics.

We pause to note that, in broad outline, these results are very similar to those obtained in the analysis of pure vibrational (bending) ${ }^{51,52}$ or rotational ${ }^{53}$ excitations of a single water molecule in neat liquid water. For all three processes, including the present case, hindered rotations are found to account for most of the excess energy transfer. But there are non-neglibible differences at the quantitative level. While for water "solute" bend excitation, transfer to rotations is overwhelming (95\%) (this includes self-rotation, see below), it diminishes (85\%) for a pure water "solute" rotational excitation, with the
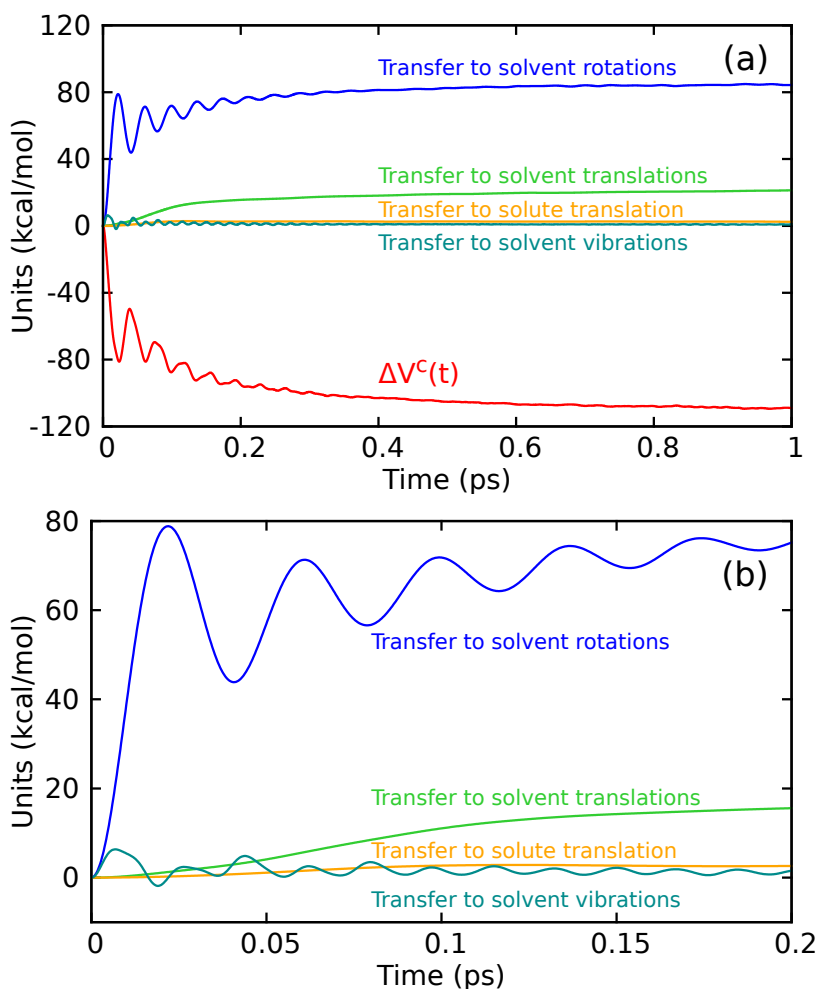

FIG. 4: Nonequilibrium relaxation functions (see Eq. 19), for positively charged ion and flexible water solvent. (a) Long time; (b) Short time.

present ion solvation coming last $(78 \%)$. Even more important differences between the solvation dynamics results and the vibrational results just cited will be taken up in Sec. IV C 2.

While the previous percentages characterize the long term approach to equilibrium, it is also of interest to examine short times, to be found in Fig. 4 (b) (the Coulomb energy decay is not included for clarity). We see that the known fast initial relaxation is possible thanks to a fast transfer into water rotations; due to the involvement of the light hydrogen atom motions, the water librations are able to respond on this short time scale to the substantial perturbation introduced by the newly created charge, and therefore dominate for all time scales. In comparison, the response of translations, governed largely by the motion of the water oxygens, is roughly an order of magnitude slower: while rotations (excluding the effect of oscillations) attain their final energy in tens of fs, translations require hundreds of fs to achieve a value close to the final equilibrium value.

Finally, we turn to the solvent stretch and bend vibrations. Although we find a small contribution from these for about $10 \mathrm{fs}$, their role results to be negligible thereafter. The very minimal energy flow to these vibrations is consistent with the lack of high frequency components of a step function transition to the charged solute state (we do not find a more significant contribution of the water bend suggested by a tcf investigation ${ }^{56}$ ). The very low 
efficiency of this channel is reassuring for our application of a classical computation, and suggests the use of rigid molecules.

\section{Rigid molecules \\ 1. Total energy fluxes}

Fig. 5 (a) displays the corresponding results for creation of a positive, unit charge in water, this time for a rigid water model (corresponding to an average over 5000 trajectories, each $2.5 \mathrm{ps}$ long). The large similarity to Fig 4(a) is confirmed by a more quantitative comparison: the percentages of energy going into rotational and translational channels do not change within the level of accuracy previously used. This is consistent with the fact that less than $1 \%$ of the energy flow went into the water bend and stretch vibrations for a flexible model, further validating the use of a rigid model.
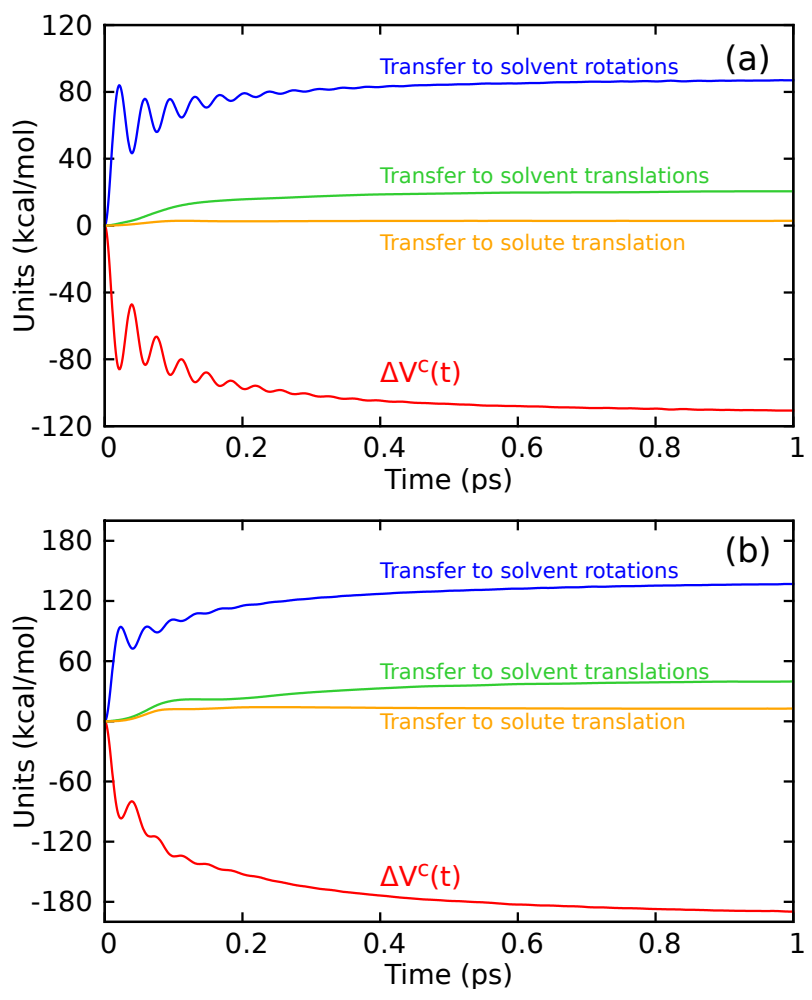

FIG. 5: Nonequilibrium relaxation functions (see Eq. 15) for rigid water solvent. (a) Produced ion with unit positive charge; (b) Same for negative charge. (Note the different vertical scales).

Panel (b) in Figure 5 corresponds to the creation of a unit negative charge rather than a positive one. It should be noted at the outset that the final equilibrium is considerably different. While for a positive charge the Coulomb energy change $\left(\Delta V^{c}\right)$ tends to $-112 \mathrm{kcal} / \mathrm{mol}$, now it exceeds $-194 \mathrm{kcal} / \mathrm{mol}$; for the particular model we use (characterized by identical LJ parameters) the hydration structure response is substantially stronger energetically ( $75 \%$ in absolute value) for the negative ion. In addition, the oscillatory behavior at short times is more damped in this case. Despite these differences, the resulting behavior for the contributions of each mode is remarkably similar. Rotations are again the leading channel with a contribution of $72 \%$, followed by $21.5 \%$ to solvent translations, and $6.5 \%$ to ion translations. These detailed energy flow results extend considerably the previous finding of Tran and Schwarz ${ }^{56}$ of the considerable similarity of the positive and negative charge tcfs (Eq. 7). The energy transfer similarity found here suggests that we might dispense with the study of one of the ions, but in fact the comparison between a positive and a negative ion will constitute an important side theme of this work, and we return to it in sub-Section IV C 2 below. Our rationale is that any electronic excitation of a solute entails the redistribution of charge; the understanding of the similarities/differences introduced by the creation of a positive/negative charge might thus provide considerable insight for more complex aqueous solvation cases.

\section{Fluxes into hydration shells}

We have noted in the preceding the similarity of the overall energy transfer patterns into water solvent modes for the present solvent dynamics case and previous studies of a vibrationally ${ }^{51,52}$ and rotationally ${ }^{53}$ excited "solute" water molecule in water. But so far we have not discussed an important detail, which is the pattern of energy flow into the hydration shells.

In order to provide a perspective for the solvation dynamics results to be presented, we first discuss in an extended fashion some further details of the prior work on energy flow from (separately) vibrationally ${ }^{51,52,76}$ and rotationally ${ }^{53}$ excited "solute" water molecule in water. The mechanism of the $\mathrm{H}_{2} \mathrm{O}$ bend vibrational relaxation is dominated by energy flow to the hindered rotation of the bend excited water molecule. This transfer, which is of course quite local, represents approximately $3 / 5$ of the transferred energy and arises from a 2:1 Fermi resonance for the centrifugal coupling between the water bend and rotation. The remaining energy flow $(\sim 2 / 5)$ from the excited water bend is directly transferred to the excited water molecule's neighbors. Approximately $2 / 3$ of this energy flow is accounted for by the first hydration shell, with a prevalence of the two water molecules H-bonded to the central oxygen. Taking the intramolecular and intermolecular routes together, we see that the first stage is a transfer of $\sim 87 \%$ of the initial energy into molecules within the first shell. Thus the initial energy flow is local, i.e. all within the first hydration shell, but it involves a number of water molecules. And since these waters receiving the energy are all H-bonded to each other, clearly some sort of "cooperative" —or "collective" — response is clearly indicated. Indeed solvation dynamics has often 
been described as collective in water ${ }^{46,56}$ (and other polar solvents $\left.{ }^{12,49,65}\right)$; an important consequence is that the overall time decay of the $\operatorname{tcf} C(t)$ is much faster than the orientation relaxation of a single water molecule. But the detailed characterization of that cooperativity or collective character has presented and still presents a fundamental problem. As was stressed earlier ${ }^{76}$, such a characterization could be provided via a representation of how the molecular perturbations just recounted could be expressed in terms of an initial excitation of e.g. water librational modes. Unfortunately, this is a route that cannot yet be followed, since there is no available simple characterization of those modes. This is an issue that will arise several times, although we attempt not to be repetitive. The energy flow from the produced rotationally excited central water molecule was characterized in detail as well ${ }^{53}$. It is less local in character than that directly from the bend to the first hydration shell: approximately $80 \%$ of its rotational kinetic energy being transferred to water molecules in the first shell, with an even sharing by molecules accepting or donating hydrogen bonds. Again, we have molecularly specific flows, but a number of coupled waters are involved and collective behavior should thereby be generated by these flows. Thus, the considerable energy flow into water librations in both the cases just described strongly suggests that the subsequent process could be described as "collective", but no quantitative description exist; an approximate field theory for librational modes might be of use here ${ }^{77}$. We can only provide an approximate characterization for the previous studies: the process is largely local (often predominantly the first hydration shell, but involving the water molecules within that shell), which will generate a collective response in the water solvent.

Returning to the present solvation dynamics case with its charge acquiring solute, we also characterize the extent to which the solvation relaxation is local or non-local and address the issue of its collective character. Certainly the long-ranged Coulomb potential for the excited state charged solute could well favor a non-local process. To begin to address these issues, we define hydration layers around the solute, so that we can compare the transfer to different layers in quantitative terms. To be useful for comparison, these definitions need to accommodate the very different nature of hydration for a neutral and a charged monoatomic solute, which can be easily seen in Fig. 6(a). This displays the radial distribution functions for the solute-water center of mass pairs (computed in separate equilibrium simulations for both neutral and charged solutes). As is to be expected (see e.g. Refs. 55,57 ), a much stronger structure exists for the ions (compared to the neutral atom), with the first peak being substantially higher and at a shorter distance from the solute center. In fact, there is almost a linear gradation (in terms of first peak height and position) in which structure increases in the sequence: neutral, positive, negative (the order for the charged cases will generally depend on the charge density, which in this case is the same).
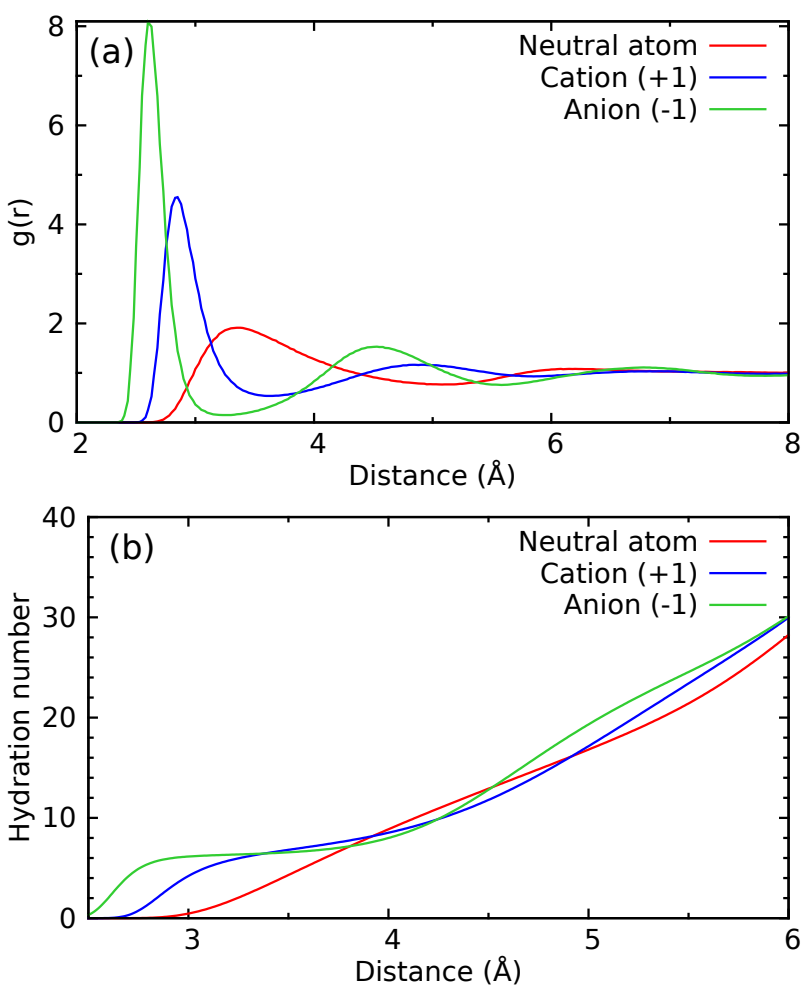

FIG. 6: Equilibrium structure: (a) Radial distribution function $(\mathrm{g}(\mathrm{r}))$ for atom-water center of mass; (b) Hydration number resulting from integration of the previous function.

For the definition of hydration layers it is also of interest to examine the running hydration number, computed from integration of the radial distribution function, and displayed in Fig. 6(b) (note that the horizontal range differs between both panels). The curves for a neutral and positively charged monoatomic cross at a distance of $3.9 \AA$, i.e. the solvation shell thus defined contains, on the average, the same number of molecules (aprox. 8) for both a positively charged and uncharged solute. Since, in addition, this distance is close to the first minimum for the cation case, it seems reasonable to select it as defining the first shell. This choice guarantees that the average number of molecules in this shell is the same at all times, so that the increase/decrease of transfer into this layer does not stem (on average) from a corresponding increase/decrease in number of molecules. We adopt the same definitions for the anion, since the scenario is quite similar (see Fig. 6(b)). For the definition of the second shell radius, we take the second minimum of the cation's radial distribution function, i.e. 6 . $\AA$, a distance at which the hydration number is identical for both the anion and cation. Both first and second shells taken together amount to roughly thirty solvent molecules (see Fig. 6(b)).

We now turn to the energy flows into each hydration shell. Fig 7(a) displays the energy flux participation of each shell for the cation (transfer into solute translations are not included). Two thirds $(67 \%)$ of the energy goes 

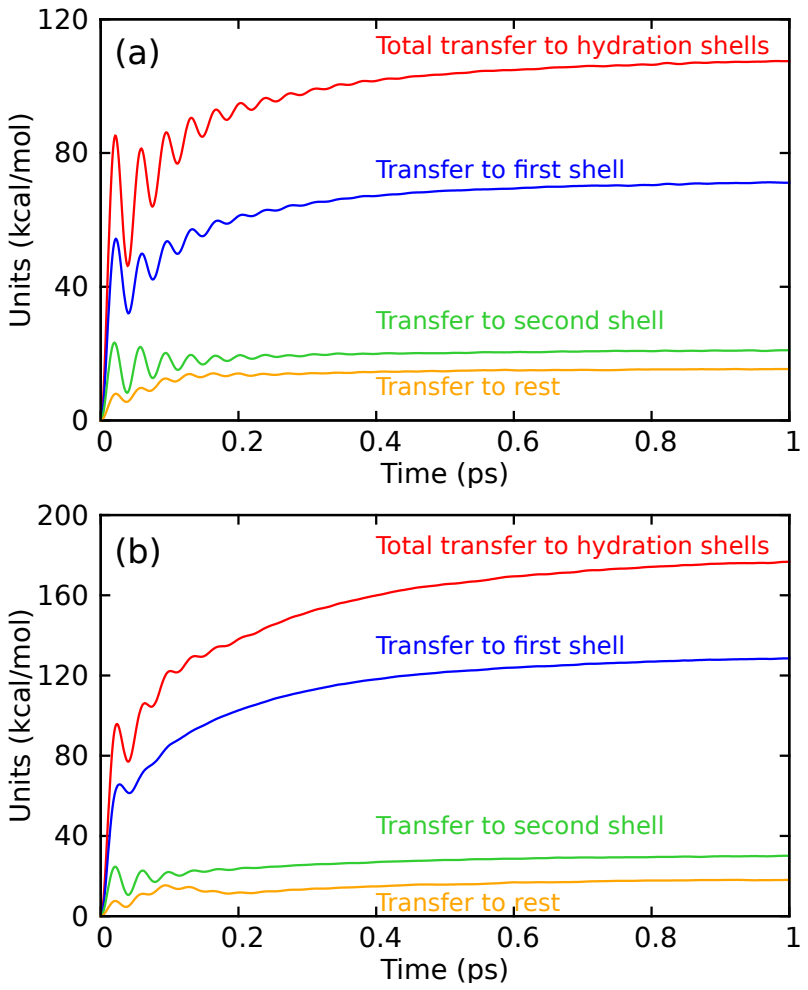

FIG. 7: Work on the different hydration shells surrounding the central ion. (a) Cation with unit charge; (b) Anion with unit charge.

into the eight molecules that constitute the first shell, while the second shell contribution amounts to $19 \%$, and that of the rest of the molecules is $14 \%$. Figure 7(b) recounts the flows for the anion, again with a similar behavior. The respective figures are: $72 \%$ (first shell), $17 \%$ (second shell) and $11 \%$ (rest). There is only a slight increase, if any, in the local character of the process for the negative ion, with a larger participation of the first shell. This can be understood as a result of the anions more pronounced first shell; this produces a shielding effect for water molecules in the second (and subsequent) shells, which are thus less affected than for the cation. In any event, in each case the first shell has $\sim 8$ waters and the energy flow to the second shell is nonnegligible, so that many waters are rotationally excited. Again, since the waters are all H-bonded to each other, collective motion is to be expected. We can gain an impression of events by examining in Fig. 8(a) the power for the 8 water molecules in the first hydration shell of a positive ion. The in-phase excitation of these waters is clear, as is the gradual dephasing of them due to interaction (and perhaps anharmonicity). The power is both positive and negative, so that the net transfer is due to the imbalance between them, a feature we previously observed in the study of the water bend relaxation ${ }^{52}$. (We will strongly contrast this phase behavior with that of the translational motions in Sec. IV C 4).

We can also point out an important aspect of the rota-
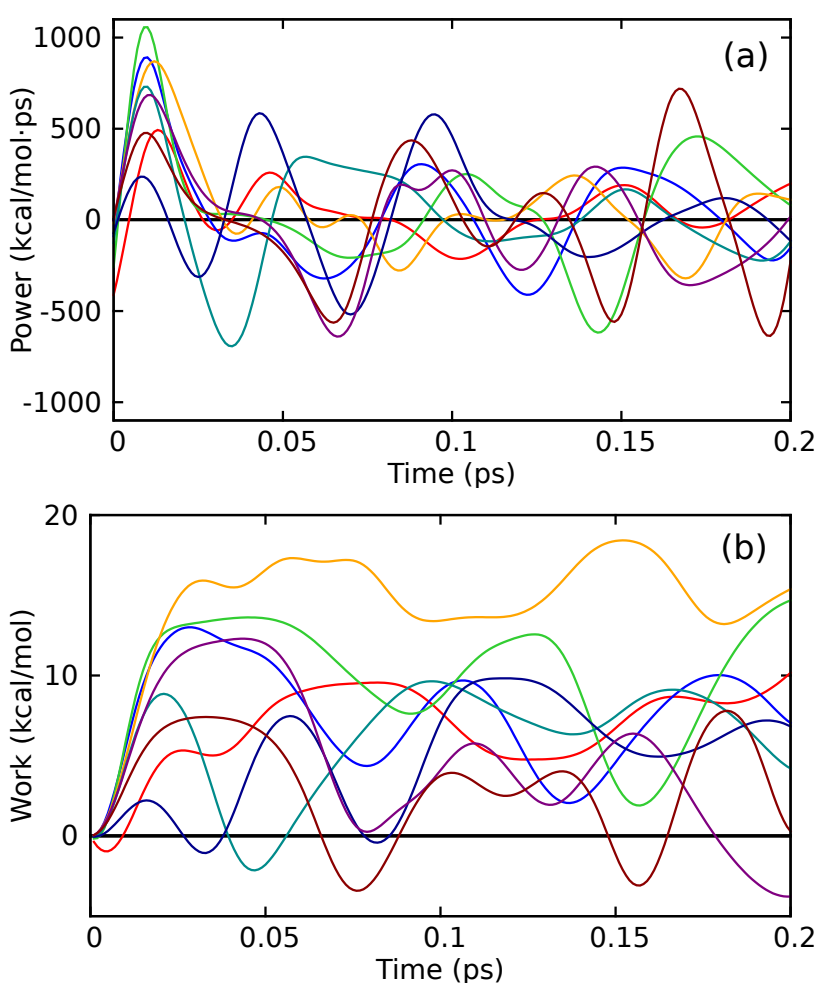

FIG. 8: (a) Power on rotations of the eight closest molecules that constitute the first shell of a newly created positive ion; (b) Work on rotations of the same water molecules.

tional energy flow by examining Fig. 8(b) which displays the work on rotation resulting from time integration of the curves in panel (a) of the same figure. The key point here is that evidently a large range, and a large amount, from a few $\mathrm{kcal} / \mathrm{mol}$ to a few tens of $\mathrm{kcal} / \mathrm{mol}$ can be channeled through the individual water molecules. The coupling between the molecules leads to a rapid passage of the solvation energy (in this case $\sim 100 \mathrm{kcal} / \mathrm{mol}$; see Fig. 5(a)). Here we can draw a parallel with the results for pure rotational excitation in Ref. 53: there it was found that rotational transfer is extermely rapid, so that energy does not sit on any water molecule for long; in addition, rotational excitations of up to $15 \mathrm{kcal} / \mathrm{mol}$ did not produce any noticeable changes in mechanism (in terms of percentages of energy flowing into the different modes).

Comparison with energy flows into surrounding hydration shells subsequent to a "solute" water bending 51,52 and rotational ${ }^{53}$ excitation is again of interest. While there the first shell is certainly the common main recipient, there are noticeable quantitative differences: for rotational relaxation, $80 \%$ of the energy is transferred to the first shell; for bending relaxation intramolecular transfer to self-rotation accounts for a $60 \%$ and transfer to first shell neighbors accounts for an additional $27 \%$ (so a total of $87 \%$ of the energy is transferred to the first shell, which now includes the initially vibrationally excited molecule). It is crucial to note that, for both those 
cases, the first shell is substantially smaller in number than for the present study (since for the water "solute" situation, this shell is defined to consist of just the four closest hydrogen bonded molecules, a choice mandated by the tetrahedral structure in the neat liquid). In addition, molecules beyond two molecular diameters barely contribute to the total relaxation, while in the present solvent dynamics study, we see that they account for a sizable portion. In short, compared to water "solute" bending and rotational energy flow, the process is clearly less local, involving a substantial number of water solvent molecules. This is consistent with the long range Coulombic perturbation introduced by the appearance of a charge, as opposed to a rotationally/vibrationally excited (neutral) water molecule which has not undergone any substantial charge redistribution.

\section{Rotational components}

We now turn to a more detailed study of the flow to the hindered rotations, focusing on the contributions from the three principal axes. Even though we have already discussed the participation of each hydration shell, we will nevertheless pay attention to their role in connection with the axes. We start with a newly created unit charge cation within our rigid water solvent model. Each panel in Fig. 9 corresponds to the first and second shells respectively (note the different vertical scales in both plots; it should be kept in mind that the second shell contribution is a minority one).

As was to be expected, transfer into the first hydration shell dominates. Since this feature has been already discussed in detail for the total work in the previous section, we will just focus on quantitative results. In particular, we find that $52 \%$ of the total initial excess energy is transferred into water librations in the first shell, i.e. half of the initial energy is directly channeled into hindered rotations of the closest eight water molecules. The novel feature here is that this transfer is clearly not evenly distributed over the water molecules' three principal axes. There is a marked dominance of transfer into rotations around the $x$ axis (see Fig. 1). This leading role even extends into the second shell (Fig. 9(b)), with the same uneven sharing among the three axes.

The (dominant) $x$ axis is characterized by the lowest moment of inertia (see Eq. 17), and it is tempting to assume that solely moment of inertia magnitude or rotational frequency considerations could guide our intuition. However there is no monotonic trend: independently of the shell considered, the axis with the largest moment of inertia ( $z$ ) comes second (rather than last), while that with the intermediate value $(y)$ is characterized by the lowest total amount of energy transfer. This seems to militate against any simple exclusively frequency-based explanation in terms of energy flowing more easily to higher frequency motions (lower moment of inertia). Indeed, we have already seen that water vibrations (char-
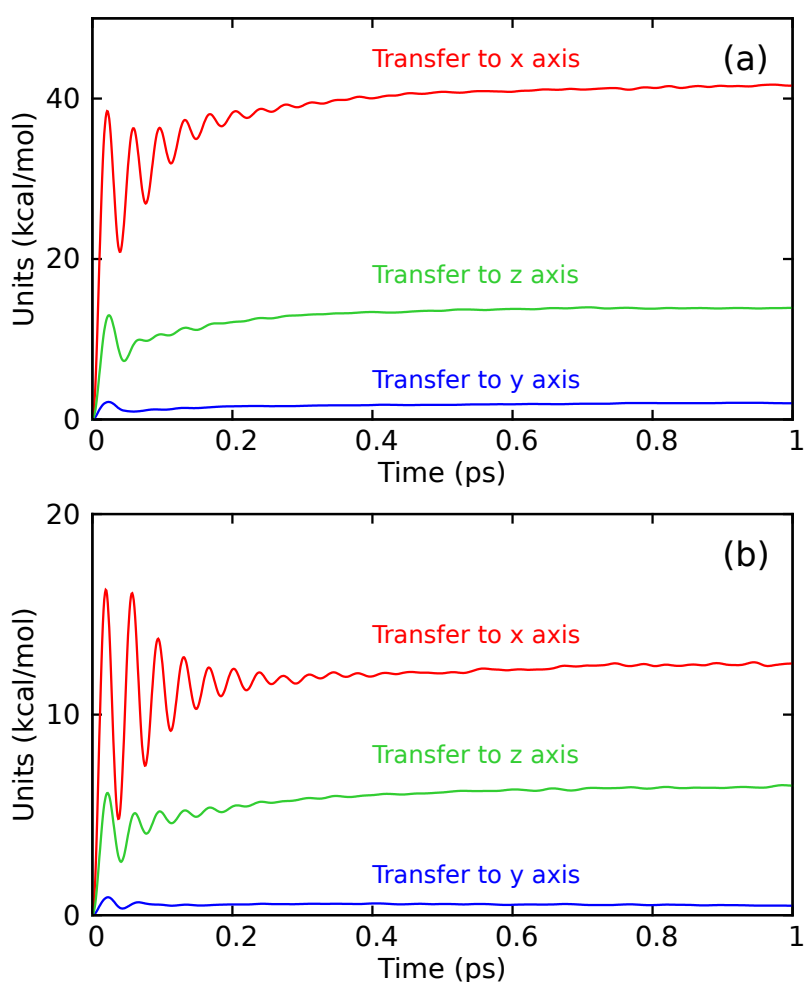

FIG. 9: Work on rotation for the water molecule principal axes for a cation. (a) First hydration shell; (b) Second shell.

acterized by much shorter time scales), do not contribute appreciably either, while translations (with their long time scales) have a secondary role as well. Clearly coupling considerations are important as well.

We can ask at this point ask to what extent this different axis contribution is altered for a negative charge. Figure 10 shows that the behavior is rather similar; the only significant change is that the $x$ axis role for second shell molecules is even more marked, at the expense of the $y$ axis. Although this similarity is in line with that discussed in the previous sub-Section for hydration shell participation, it is nevertheless surprising. The orientations of the water molecules around positive and negative ions are dramatically different due to water's highly directional hydrogen bonding. The comparison of radial distribution functions in Fig. 6(a) clearly indicates this, but representative configurations might be more illustrative. Figure 11 displays instantaneous configurations for the central atom and first hydration shell for each of the three cases (neutral atom, anion, cation).

For the neutral atom, the surrounding water molecules form a sort of hydrogen-bonded chain that surrounds the atom quite unevenly. For the charged case, though, molecules are more uniformly distributed, but with substantially different orientations, as is well known (see e.g. Refs. 55,57). There is a relatively tight structure for the anion, in which one hydrogen from each water molecule is closest to the central negative charge. For the cation, 

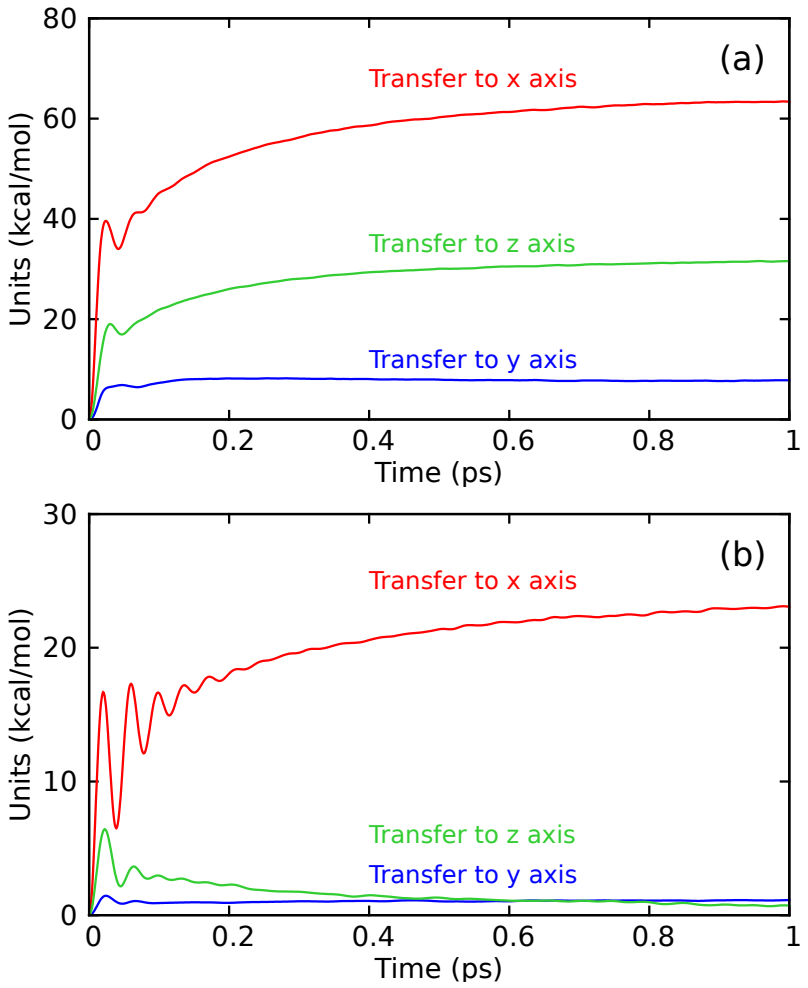

FIG. 10: Work on rotation for the water molecule principal axis for an anion. (a) First hydration shell; (b) Second shell.

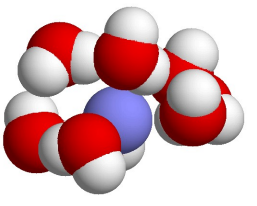

neutral

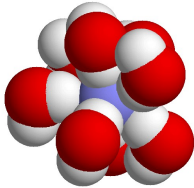

anion

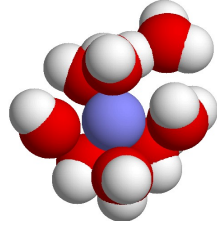

cation
FIG. 11: Snapshots of the closest eight water molecules and central solute (blue).

the structure is not so marked (for the present model in which cation and anion have the same LJ radius), but now it is of course the oxygen center that is closest to the central atom. Given these structural differences the similar water axis patterns for energy flow into librations for oppositely charged solutes is remarkable and we return to this issue in the last Section.

\section{Translational components}

The first remark is to recall, e.g. from Fig. 5, that the translational component of the relaxation is both smaller in extent and more slowly evolving than is the rotational contribution ${ }^{78}$. This disparity is also reflected in the power and work associated with the first hydration shell water molecules in Fig. 8 and Fig. 12 respectively.
Before entering into assorted further details, we immediately point out an additional key difference in the nature of the energy transfer compared to the behavior of first shell waters in the rotational case: Figure 12(a) does not show the in-phase excitation behavior for the rotational case that was apparent in Fig. 8(a).
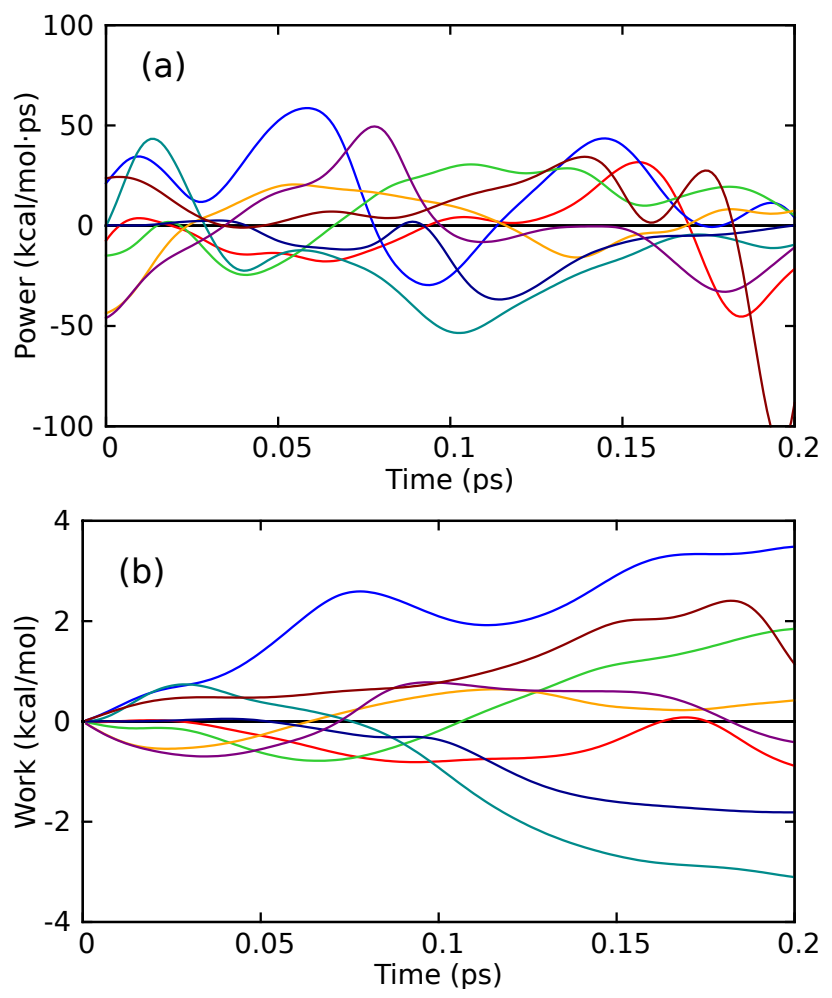

FIG. 12: (a) Power on translations of the eight closest molecules that constitute the first shell of a newly created positive ion; (b) Work on translations of the same water molecules.

We also need to recall that besides our usual partition into shells, a partition for translation into isotropic and anisotropic contributions is also performed, as explained in Sec. IIB. The center of mass velocity is split into its parallel and perpendicular components with respect to the ion-water center of mass. Power exerted on the parallel component is denoted as isotropic, while that exerted on the perpendicular component is defined as anisotropic, in analogy to the definitions adopted in the original experimental work ${ }^{69}$.

Fig. 13 summarizes the results for a positive ion. For the partition into hydration shells (panel (a)), we find again the dominance of transfer into the first shell, which accounts for $67 \%$. Another $8 \%$ is transferred into the second shell, while the rest of the water solvent takes $25 \%$; similar results are obtained for an anion, see Fig. 14. These figures do not differ significantly from those that correspond to the total works on each shell (see Figs. 7 ), except that now the contribution from the second shell is the smallest. The higher proportion now transferred into water molecules beyond the second shell signals that 

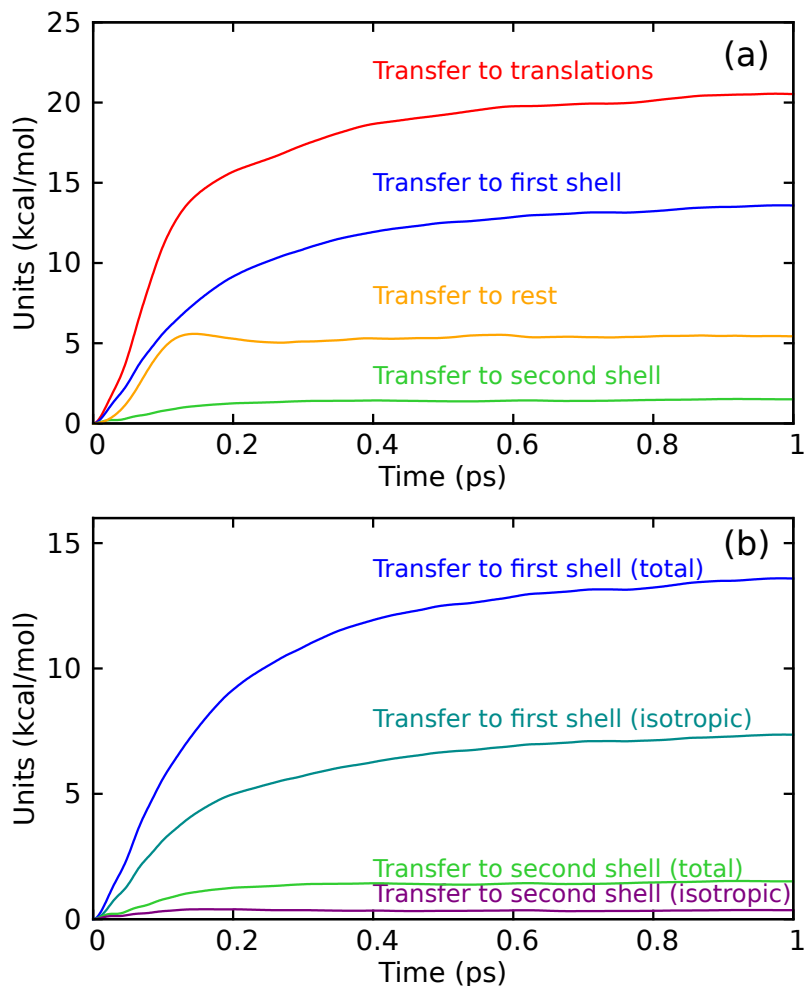

FIG. 13: Work on solvent translations for a cation. (a) Partitioned into hydration shells; (b) Showing the isotropic component for the first two shells.

this process might be slightly more non-local than that of the rotational transfer. It should be mentioned though that, for this particular function, a computation with a flexible model renders the two minority contributions (second shell vs. the rest of molecules) closer to each other, so that it is evidently a minor effect.

As for the isotropy of the transfer into translations, the isotropic part (Fig. 13(b)) accounts for half (53\%) of the work within the first shell, and has a minor contribution to the second shell $(13 \%)$. Here we find a difference with the negative charge case (Fig. 14(b)), where the isotropic part is dominant for both shells $(88 \%$ for the first shell, and $70 \%$ for the second shell). To gain some perspective on these results, we have computed a related quantity in the ideal case of an ion in the presence of single water molecule with random orientation. For each configuration, we have computed the total force modulus and compared it to the modulus of the component along the ion-water molecule axis (which we associate with the isotropic component), averaging over a set of random orientations. The result is independent of the sign of the ion charge, and the isotropic part accounts for $79 \%$ of the total modulus. Taking this result as a reference, we see that for the ion in the neat liquid, a shift in opposite directions occurs, depending on the sign of the ion's charge: less for the cation and greater for the anion. In short, the behavior for transfer into translation is somewhat more sensitive to the excite state solute's charge sign than that
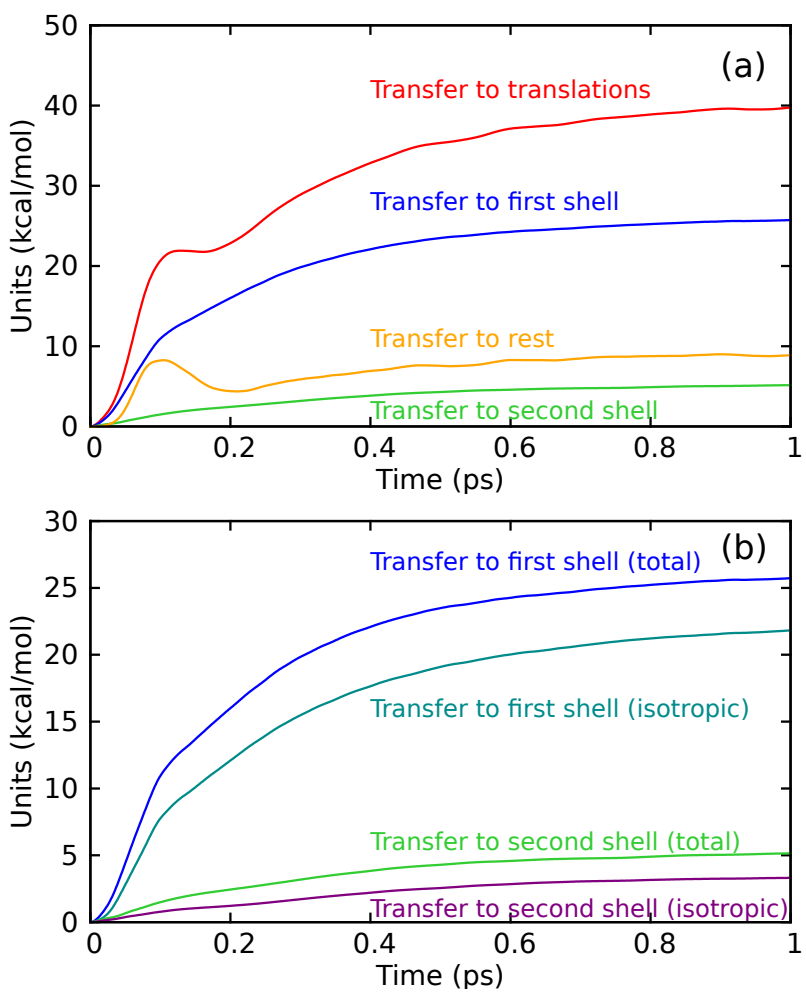

FIG. 14: Work on solvent translations for an anion. (a) Partitioned into hydration shells; (b) Showing isotropic component for the first two shells. For a given shell, the anisotropic part is of course the difference of this form the total.

of transfer into rotations.

\section{CONCLUDING REMARKS}

We start by summarizing the main characteristics of the solvation dynamics process - viewed from the present energy flow perspective - for the sudden change of a neutral solute to an excited state positively or negatively charged solute in aqueous solution. First, it is fairly nonlocal, involving tens of water solvent molecules, although the first shell (eight molecules) accounts for fully half of the energy transfer. Due to the strong hydrogen bonding interactions between the water molecules, this will lead to some type of collective or cooperative motions. Second, hindered rotations (librations) are by far the main recipient of the initial excess energy, a channel which exhibits initial in-phase motion of the nearest water moleculesa behavior not exhibited by the water translations - and this phasing disappears due to the interactions between the waters. This rotational channel, which very rapidly transports energy through the hydration shells without any one gaining an excessive amount of energy, is dominated by water rotations around the axis with the lowest moment of inertia. Third, the previous points hold for both positive and negative charges, a feature which is rather intriguing, considering that the final structure of 
the first shell, due to the directional nature of hydrogen bonding, is radically different for a positive and a negative ion.

From a more general standpoint, the computation of detailed energy fluxes seems to hold considerable potential in order to achieve a better molecular level understanding of solvation relaxation. The case of a diatomic undergoing a change of dipole moment could prove a particularly natural choice (and one reflective of a less concentrated charge redistribution), but we consider that this methodology should also be adequate and specially useful for the challenge of increasing levels of complexity where a molecular perspective has been particularly difficult to achieve ${ }^{18,19,84,85}$. But before addressing these issues, we first intend to report in this series on some aspects that have been left untouched for the simple solute problem addressed here. The question of the relation between energy fluxes and structural changes within the water solvent is one of them, together with a more detailed characterization of the delocalized nature of the energy flows; another is the remarkably similar water axis patterns for energy flow into librations for oppositely charged solutes despite the strong differences in their hydration shell structures. Perhaps even more importantly, we will also address the issue of the microscopic nature of the so-called "inertial" and "diffusive" regimes of solvation dynamics.

\section{Acknowledgments}

This work was supported by DGR (2009-SGR-1003) and MICINN (FIS2012-394-C02-01)(RR), and NSF grant CHE-1112564 (JTH).

Supporting Information Available: Discussion of the procedure to obtain, from the instantaneous positions and velocities of a molecule, the corresponding translational, rotational and vibrational velocities. This material is available free of charge via the Internet at http://pubs.acs.org.
* Electronic address: rosendo.rey@upc.edu

$\dagger$ Electronic address: hynes@spot.colorado.edu

1 Bagchi, B. Dynamics of Solvation and Charge-Transfer Dynamics in Liquids. Ann. Rev. Phys. Chem. 1989, 40, 115141.

2 Maroncelli, M.; Macinnis, J.; Fleming, G.R. Polar-Solvent Dynamics and Electron-Transfer Reactions. Science 1989, 243, 1674-1681.

${ }^{3}$ Fleming, G.R.; Wolynes, P.G. Chemical Dynamics in Solution. Phys. Today 1990, 43, 36-43.

${ }^{4}$ Fleming, G.R.; Cho, M.H. Chromophore-Solvent Dynamics. Ann. Rev. Phys. Chem. 47, 47, 109-134.

${ }^{5}$ Cho, M.; Fleming, G.R. Electron Transfer and Solvent Dynamics in Two- and Three-State Systems. Adv. Chem. Phys. 1999, 107, 311-370.

${ }^{6}$ Nandi, N.; Bhattacharyya, K.; Bagchi, B. Dielectric Relaxation and Solvation Dynamics of Water in Complex Chemical and Biological Systems. Chem. Rev. 2000, 100, 20132045.

7 Maroncelli, M. The Dynamics of Solvation in Polar Liquids. J. Mol. Liq. 1993, 5\%, 1-37.

8 Stratt, R.M.; Maroncelli, M. Nonreactive Dynamics in Solution: The Emerging View of Solvation Dynamics and Vibrational Relaxation. J. Phys. Chem. 1996, 100, 1298112996.

9 Bagchi, B.; Jana, B. Solvation Dynamics in Dipolar Liquids. Chem. Soc. Rev. 2010, 39, 1936-1954.

10 Maroncelli, M.; Fleming, G.T. Picosecond Solvation Dynamics of Coumarin 153: the Importance of Molecular Aspects of Solvation. J. Chem. Phys. 1987, 86, 6221-6239.

11 Jimenez, R.J.; Fleming, G.R.; Kumar, P.V.; Maroncelli, M. Femtosecond Solvation Dynamics in Water. Nature 1994, 369, 471-473.

12 Horng, M.L.; Gardecki, J.A.; Papazyan, A.; Maroncelli, M. Subpicosecond Measurements of Polar Solvation Dy- namics: Coumarin 153 Revisited. J. Phys. Chem. 1995, 99, 17311-17337.

13 Neria, E.; Nitzan, A. Numerical Simulations of Solvation Dynamics in Electrolyte Solutions. J. Chem. Phys. 1994, 100, 3855-3868.

14 Chandra, A.; Patey, G.N. Solvation Dynamics in Electrolyte Solutions. J. Chem. Phys. 1994, 100, 1552-1558.

15 Duan, J.; Shim, Y.; Kim, H.J. Solvation in Supercritical Water. J. Chem. Phys. 2006, 124, 204504.

16 Biswas, R.; Bagchi, B., Ion Solvation Dynamics in Supercritical Water. Chem. Phys. Lett. 1998, 290, 223-228.

17 Re, M.; Laria, D. Dynamics of Solvation in Supercritical Water. J. Phys. Chem. B 1997, 101, 10494-10505

18 Terranova, Z.L.; Corcelli, S.A: On the Mechanism of Solvation Dynamics in Imizazolium-Based Ionic Liquids. $J$. Phys. Chem. B 2013, 49, 15659-15666.

19 Zhang, X.X.; Liang, M.; Ernsting, N.P.; Maroncelli, M. Conductivity and Solvation Dynamics in Ionic Liquids. J. Phys. Chem. Lett. 2013, 4, 1206-1210.

20 Benjamin, I. Chemical Reactions and Solvation at Liquid Interfaces. Chem. Rev. 1996, 96, 1449-1476.

21 Michael, D.; Benjamin, I. Molecular Dynamics Computer Simulations of Solvation Dynamics at Liquid/Liquid Interfaces. J. Chem. Phys. 2001, 114, 2817-2824.

${ }^{22}$ Faeder, J.; Ladanyi, B.M. Solvation Dynamics in Reverse Micelles: The Role of Headgroup-Solute Interactions. $J$. Phys. Chem. B 2005, 109, 6732-6740.

23 Levinger, N.E.; Swafford, L.A. Ultrafast Dynamics in Reverse Micelles. Ann. Rev. Phys. Chem. 2009, 60, 385-406.

24 Rodríguez, J.; Martí, J.; Guàrdia, E.; Laria, D. Exploring the Picosecond Time Domain of the Solvation Dynamics of Coumarin 153 within Beta-Cyclodextrins. J. Phys. Chem. B 2008, 112, 8990-8998.

25 Pal, S.K.; Peon, J.; Zewail, A.H. Biological Water at the Protein Surface: Dynamical solvation Probed Directly 
with Femtosecond Resolution. Proc. Natl. Acad. Sci. USA 2002, 99, 1763-1768.

${ }^{26}$ Qiu, W.; Zhang, L.; Okobiah, O.; Yang, Y.; Wang, L.; Zhong, D.; Zewail, A.H. Ultrafast Solvation Dynamics of Human Serum Albumin: Correlations with Conformational Transitions and Site-Selected Recognition. J. Phys. Chem. B 2006, 110, 10540-10549.

27 Golosov, A.A.; Karplus, M. Probing Polar Solvation Dynamics in Proteins: A Molecular Dynamics Simulation Analysis. J. Phys. Chem. B 2007, 111, 1482-1490.

28 Zhang, L.; Wang, L.; Kao, Y.T.; Qiu, W.; Yang, Y.; Okobiah, O.; Zhong, D. Mapping Hydration Dynamics Around a Protein Surface. Proc. Natl. Acad. Sci. 2007, 104, 1846118466.

29 Zhong, D.; Pal, S.K.; Zewail, A.H. Biological Water: A Critique. Chem. Phys. Lett. 2011, 503, 1-11.

30 Yang, Y.; Qin, Y.; Ding, Q.; Bakhtina, M.; Wang, L., Tsai, M.D. Ultrafast Water Dynamics at the Interface of the Polymerase-DNA Binding Complex. Biochemistry 2014, 53, 5405?5413

31 Pal, S.; Maiti, P.K.; Bagchi, B.; Hynes, J.T. Multiple Timescales in Solvation Dynamics of DNA in Aqueous Solution: Role of Water, Counterions, and CrossCorrelations. J. Phys. Chem. B 2006, 110, 26396-26402.

32 Furse, K.E.; Corcelli, S.A. The dynamics of water at DNA interfaces: Computational Studies of Hoechst 33258 Bound to DNA. J. Am. Chem. Soc. 2008, 130, 13103-13109.

33 Sen, S.; Andreatta, D.; Ponomarev, S.Y.; Beveridge, D.L.; Berg, M.A. Dynamics of Water and Ions Near DNA: Comparison of Simulation to Time-Resolved Stokes-Shift Experiments. J. Am. Chem. Soc. 2009, 131, 1724-1735.

${ }^{34}$ K. E. Furse, K.E.; and S. A. Corcelli, S.A. Molecular Dynamics Simulations of DNA Solvation Dynamics. J. Phys. Chem. Lett. 2010, 1, 1813-1820.

35 Hynes, J.T. Outer Sphere Electron Transfer Reactions and Frequency-Dependent Friction. J. Phys. Chem. 1986, 90, 3701-3706.

${ }^{36}$ Smith, B.B.; Hynes, J.T. Electronic Friction and Electron Transfer Rates at Metallic Electrodes. J. Chem. Phys. 1993, 99, 6577-6590.

37 Borgis, D.; Hynes, J.T. Molecular Dynamics Simulation for a Model Nonadiabatic Proton Transfer Reaction in Solution. J. Chem. Phys. 1991, 94, 3619-3628.

38 Bruehl, M.; Hynes, J.T. Dielectric Friction and Solvation Dynamics: A Molecular Dynamics Study. J. Phys. Chem. 1992, 96, 4068-4074.

39 Hynes, J.T. Charge Transfer Reactions and Solvation Dynamics. In Ultrafast Spectroscopy; Simon, J., Ed.; Kluwer: Dordrecht, 1994; pp. 345-381.

40 Malhado, J.; Spezia, R.; Hynes, J.T. Dynamical Friction Effects on the Photoisomerization of a Model Protonated Schiff Base in Solution. J. Phys. Chem. A 2011, 115, 37203735 .

41 Gertner, B.J.; Whitnell, R.M.; Wilson, K.R.; Hynes, J.T. Activation to the Transition State: Reactant and Solvent Energy Flow for a Model SN2 Reaction in Water. J. Amer. Chem. Soc. 1991, 113, 74-87.

42 Oxtoby, D.W. Vibrational population relaxation in liquids. Adv. Chem. Phys. 1981, 47, 487-519.

43 Whitnell, R.M.; Wilson, K.R.; Hynes, J.T. Vibrational Relaxation of a Dipolar Molecule in Water. J. Chem. Phys. 1992, 96, 5354-5369.

${ }^{44}$ Rey, R.; Hynes, J.T. Vibrational Relaxation of HOD in $\mathrm{D}_{2} \mathrm{O}$. J. Chem. Phys. 1996, 104, 2356-2368.
45 Sibert, E.L.; Rey, R. Vibrational relaxation in liquid chloroform following ultrafast excitation of the $\mathrm{CH}$ stretch fundamental. J. Chem. Phys. 2002, 116, 237-257.

46 Maroncelli, M.; Fleming, G.R. Computer Simulation of the Dynamics of Aqueous Solvation. J. Chem. Phys. 1988, 89, 5044-5068

47 Fonseca, T.; Ladanyi, B.M. Breakdown of Linear Response for Solvation Dynamics in Methanol. J. Phys. Chem. 1991, 95, 2116-2119.

48 Aherne, D.; Tran, V.; Schwartz, B.J. Nonlinear, Nonpolar Solvation Dynamics in Water: the Roles of Electrostiction and Solvent Translation in the Breakdown of Linear Response. J. Phys. Chem. B 2000, 104, 5382-5394.

49 Bedard-Hearn, M.J.; Larsen, R.E.; Schwartz, B.J. Hidden Breakdown of Linear Response: Projections of Molecular Motions in Nonequilibrium Simulations of Solvation Dynamics. J. Phys. Chem. A 2003, 10\%, 4773-4777.

50 Carter, E.; Hynes, J.T. Solvation Dynamics for an Ion Pair in a Polar Solvent: Time-Dependent Fluorescence and Photochemical Charge Transfer. J. Chem. Phys. 1991, 94, 5961-5979.

51 Rey, R.; Hynes, J.T. Tracking Energy Transfer from Excited to Accepting Modes: Application to Water Bend Vibrational Relaxation. Phys. Chem. Chem. Phys. 2012, 14, 6332-6342.

${ }^{52}$ Rey, R.; Ingrosso, F.; Elsaesser, T.; Hynes, J.T. Pathways for $\mathrm{H}_{2} \mathrm{O}$ Bend Vibrational Relaxation in Liquid Water. $J$. Phys. Chem. A 2009, 113, 8949-8962.

53 Petersen, J.; Møller, K.B.; Rey, R.; Hynes, J.T. Ultrafast Librational Relaxation of $\mathrm{H}_{2} \mathrm{O}$ in Liquid Water. J. Phys. Chem. B 2013, 117, 4541-4552.

54 Perera, L.; Berkowitz, M.L. Dynamics of Ion Solvation in a Stockmayer Fluid. J. Chem. Phys. 1992, 96, 3092-3101.

55 Koneshan, S.; Rasaiah, J.C.; Lynden-Bell, R.M.; Lee, S.H. Solvent Structure, Dynamics, and Ion Mobility in Aqueous Solutions at 25 C. J. Phys. Chem. B 1998, 102, 4193-4204.

56 Tran, V.; Schwartz, B.J. Role of Nonpolar Forces in Aqueous Solvation: Computer Simulation Study of Solvation Dynamics in Water Following Changes in Solute Size, Shape and Charge. J. Phys. Chem. B 1999, 103, 55705580.

57 Rasaiah, J.C.; Lynden-Bell, R.M. Computer Simulation Studies of the Structure and Dynamics of Ions and NonPolar Solutes in Water. Phil. Trans. R. Soc. Lond. A 2001, 359, 1545-1574.

58 Carter, E.A.; Hynes, J.T. Solute-Dependent Solvent Force Constants for Ion Pairs and Neutral Pairs in a Polar Solvent. J. Phys. Chem. 1989, 93, 2184-2187.

59 Ladanyi, B.M.; Perng, B.C. Solvation Dynamics in Dipolar-Quadrupolar Mixtures: a Computer Simulation Study of Dipole Creation in Mixtures of Acetonitrile and Benzene. J. Phys. Chem. A 2002, 106, 6922-6934.

${ }^{60}$ In other more complex cases, while the procedure will not be so straightforward, the power/work formulation can be equally applied.

61 Roy, S.; Bagchi, B. Solvation Dynamics in Liquid Water. A Novel Interplay between Librational and Diffusive Modes. J. Chem. Phys. 1993, 99, 9938-9943.

${ }^{62}$ Nandi, N.; Roy, S.; Bagchi, B. Ionic and Dipolar Solvation Dynamics in Liquid Water. Proc. Indian Acad. Sci. (Chem. Sci.) 1994, 106, 1297-1306.

63 Nandi, N.; Roy, S.; Bagchi, B. Ultrafast Solvation Dynamics in Water: Isotope Effects and Comparison with Experimental Results. J. Chem. Phys. 1995, 102, 1390-1397. 
${ }^{64}$ Our work is most closely related to, but differs from (see especially Section II), that of Ladanyi and Maroncelli ${ }^{65}$ in which they use Steele theory ${ }^{66}$, which analyzes the role of molecular degrees of freedom by investigating the tcf of cross products of energy gap derivatives, and particularly to the extension by Schwartz and col. ${ }^{49,67}$ to nonequilibrium simulations of solvation in liquid tetrahydrofuran.

${ }^{65}$ Ladanyi, B.M.; Maroncelli, M. Mechanisms of Solvation Dynamics of Polyatomic Solutes in Polar and Nondipolar Solvents: a Simulation Study. J. Chem. Phys. 1998, 109, 3204-3221.

66 Steele, W. A Theoretical Approach to the Calculation of Time-Correlation Functions of Several Variables. Mol. Phys., 1987, 61, 1031-1043.

67 Bedard-Hearn, M.J.; Larsen, R.E.; Schwartz, B.J. Understanding Nonequilibrium Solute and Solvent Motions through Molecular Projections: Computer Simulations of Solvation Dynamics in Liquid Tetradhydrofuran (THF). $J$. Phys. Chem. B, 2003, 10\%, 14464-14475.

68 Ladanyi, B.M.; Stratt, R.M. Short Time Dynamics of Vibrational Relaxation in Molecular Fluids. J. Phys. Chem. A 1998, 102, 1068-1082.

69 Park, S.; Kim, J.; Moran, A.M.; Scherer, N.F. Solvent Structural Relaxation Dynamics in Dipolar Solvation Studied by Resonant Pump Polarizability Response Spectroscopy. Phys. Chem. Chem. Phys. 2011, 13, 214-223.

70 Bright Wilson, E.; Decius, J.C.; Cross, P.C. Molecular Vibrations; Dover: New York, 1980.

71 Berendsen, H. J. C.; Grigera, J.R.; Straatsma, T.P. The Missing Term in Effective Pair Potentials. J. Phys. Chem 1987, 91, 6269-6271.

72 Toukan, K.; Rahman, A. Molecular Dynamics Study of Atomic Motions in Water. Phys. Rev. B 1985, 31, 26432648.

73 Berendsen, H.J.C.; Postma, J.P.M.; van Gunsteren, W.F.; DiNola, A.; Haak, J.R. Molecular Dynamics with Coupling to an External Bath. J. Chem. Phys. 1984, 81, 3684-3690.

74 The term "inertial" has been used in two quite different senses in the solvation dynamics literature. In Ref. 50, Carter and Hynes employed the term to refer exclusively to the initial Gaussian time dependent component of the spectral dynamics, in which forces change due to free streaming, i.e. inertial motion of the solvent molecules (i.e. their motion in this context is not influenced by forces or torques). In many other discussions of solvation dynamics, "inertial" is used in a much more general sense to simply indicate short time dynamics. For example, rapid spectral dynamics due to librational motions in water would be called "inertial" in this latter sense, even though these solvent molecule motions are by definition strongly influenced by torques and display non-Gaussian dynamics (and are thus not "inertial" in the former sense).

75 Model theoretical studies ${ }^{61-63}$ on a similar but larger radius ionic solute system in water assign somewhat more importance to solvent translations, as noted at the beginning of Sec. IV C4 in Ref. 78

${ }^{76}$ Ingrosso, F.; Rey, R.; Elsaesser, T.; Hynes, J.T. Ultrafast Energy Transfer from the Intramolecular Bending Vibration to Librations in Liquid Water. J. Phys. Chem. A 2009, 113, 6657-6665.

77 Something in the overdamped limit of particle reoriention already exists, but requires extension to the inclusion of inertia to more directly connect to librations: Hynes, J.T.; Kapral, R.; Weinberg, M. Particle Rotation and Transla- tion in a Fluid with Spin. Physica 1977, 87A, 427-452.

78 Model theoretical studies of the water solvation dynamics for a larger ionic solute and based on a Molecular Hydrodynamics approach ${ }^{61-63}$ attribute a more significant role to water translational motion on the shortest time scale than we find (compare Fig. 1 of Ref 61 with Fig. 5 of the present work). In this connection, an important role is assigned in Refs. 61-63 to the oxygen-oxygen hydrogen bonding mode at $\sim 200 \mathrm{~cm}^{-1}$. This mode was originally thought to require the explicit inclusion of polarizability fo its existence ${ }^{79,80}$, but this turned out not to be the case but instead to be water model-dependent ${ }^{81-83}$. In particular, for the non-polarizable SPCE model for water employed in the present work, this mode appears to be welldescribed ${ }^{82,83}$. Whether the difference in the importance of solvent translational motion is due to the Molecular Dynamics theoretical approach, the different solute characteristics, solvent polarizability, or some other reason is an interesting issue worth some examination.

79 Impey, R.W.; Madden, P.A.; McDonald, I.R. OxygenOxygen Correlations in Liquid Water. Chem. Phys. Lett. 1982, 88, 589-594.

80 Neumann, M. Dielectric Relaxation in Water - Computer Simulations with the TIP4P Potential. J. Chem. Phys. 1986, 85, 1567-1580.

81 Martí, J.; Padró, J.A.; Guàrdia, E. Molecular Dynamics Simulation of Liquid Water along the Coexistence Curve: Hydrogen Bonds and Vibrational Spectra. J. Chem. Phys. 1996, 105, 639-649.

${ }^{82}$ Rey, R.; Møller, K.B.; Hynes, J.T. Hydrogen Bond Dynamics in Water and Ultrafast Infrared Spectroscopy. $J$. Phys. Chem. A 2002, 106, 11993-11996.

83 Møller, K.B.; Rey, R.; Hynes, J.T. Hydrogen Bond Dynamics in Water and Ultrafast Infrared Spectroscopy: A Theoretical Study. J. Phys. Chem. A 2004, 108, 12751289.

${ }^{84}$ Roy, S.; Bagchi, B. Solvation Dynamics of Tryptophan in Water-Dimethyl Sulfoxide Binary Mixture: in Search of Molecular Origin of Composition Dependent Multiple Anomalies. J. Chem. Phys. 2013, 139, 034308.

85 Nishiyama, K.; Watanabe, Y.; Yoshida, N.; Hirata, F. Solvent Dependence of Stokes Shift for Organic Solute-Solvent Systems: a Comparative Study by Spectroscopy and Reference Interaction-Site Model-Self-Consistent-Field Theory. J. Chem. Phys. 2013, 139, 094503. 
TOC figure

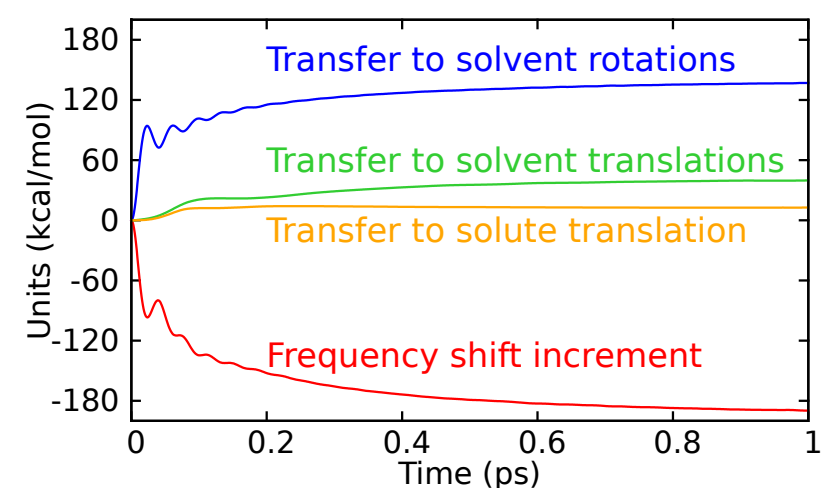

